\title{
LA PIEDRA HINCADA DE EL SHINCAL DE QUIMIVIL
}

\section{PIEDRA HINCADA OF EL SHINCAL DE QUIMIVIL}

\author{
Reinaldo Andrés Moralejo \\ CONICET. División Arqueología, Museo de La Plata. FCNyM, UNLP \\ E-mail: reinaldomoralejo@yahoo.com.ar
}

Presentado el: 27/09/2012 - Aceptado 27/02/2013

\section{Introducción}

Las investigaciones de los sitios incaicos implican un cierto abordaje teórico-metodológico en pos de caracterizar y comprender diferentes aspectos de la vida cotidiana, social, política, ritual y económica de una sociedad, en este caso la incaica. El objetivo de este trabajo consiste en la presentación y caracterización de una kancha incaica localizada a la vera de la vía principal de acceso al sitio El Shincal de Quimivil, ubicado en la localidad de Londres del departamento de Belén, en el centro-oeste de la provincia de Catamarca. Dicha vía constituye un tramo del Qhapaq Ñan o Camino Principal Andino que ha sido identificado como el "camino de la Sierra" de acuerdo al cronista Antonio de Herrera y Tordesillas (1492-1531) (Raffino 1981; Hyslop 1984).

Este conjunto residencial se encuentra ubicado en el sector norte del sitio El Shincal de Quimivil y ha sido denominado Conjunto Piedra Hincada (Figura 1). El mismo fue trabajado por primera vez, hacia fines de la década del '90, por el Dr. Rodolfo Raffino y un equipo de colaboradores conformado por los Lics. Darío Iturriza, Diego Gobbo y Victoria Montes. Después de realizar excavaciones en el interior de dos recintos, los materiales obtenidos quedaron depositados, durante un largo tiempo, en el Museo El Shincal de la localidad de Londres sin ser analizados. Con motivo de emprender nuestras investigaciones, vinculadas a las unidades residenciales asociadas al Qhapaq $\tilde{N} a n^{1}$, decidimos dar con dichos materiales y proceder a su respectivo análisis. Esta tarea también trajo aparejado recabar el resto de la información como libretas de campo y planillas de excavación. También se realizaron nuevos trabajos de prospección y análisis arquitectónico y topográfico. En cuanto a los materiales recuperados, lamentablemente, y por causas desconocidas, solo se pudo dar con ellos de manera parcial. 


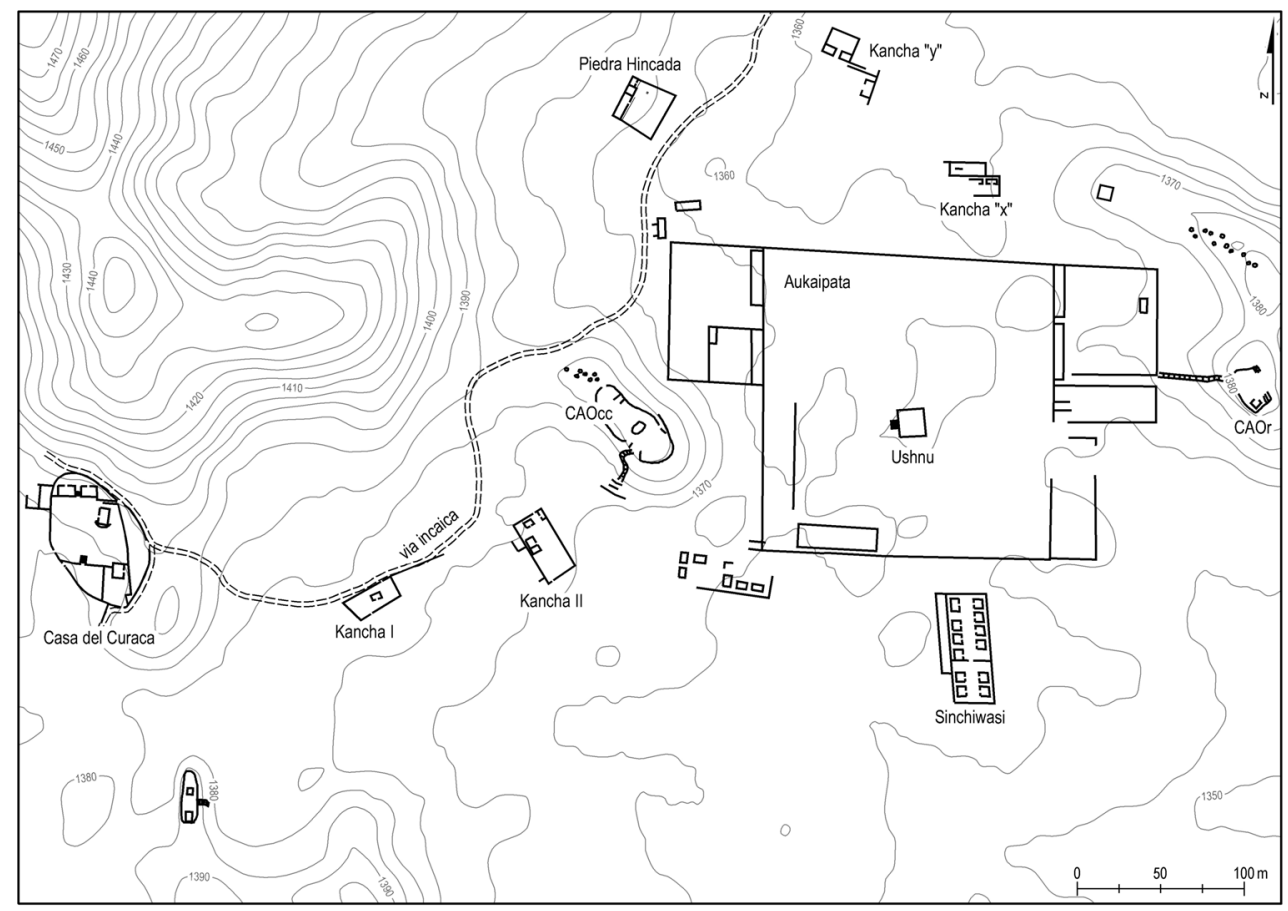

Figura 1. Pl Figura 1. Plano del sitio El Shincal de Quimivil (CAOc. = Cerro Aterrazado Occidental; CAOr.= Cerro Aterrazado Oriental) (tomado y modificado de Raffino et al. 1982, lámina 4 y Farrington 1999:62)

\section{El vocablo kancha}

El termino kancha es una palabra quechua que tiene varias acepciones según el contexto en el que aparece asociada, pero siempre hace referencia a la organización andina del espacio. Es por ello que posee un uso cotidiano con una amplia connotación social, política y religiosa (Matos Mendieta 1994). Una kancha constituye un conjunto de casas ortogonales, ordenadas en torno a un espacio central, encerradas por un muro de planta, también ortogonal, dispuesta a manera de muro perimetral, con una o dos puertas de acceso (Gasparini y Margolies 1977; Hyslop 1990; Matos Mendieta 1994). Existen, también, algunos casos de kancha de planta circular, muy comunes en la sierra norcentral del Perú (Herrera 2004).

Según Madrazo y Ottonello de García Reinoso (1965), desde el punto de vista arquitectónico, estas unidades se corresponden con la definición de Rectángulo Perimetral Compuesto (RPC); y si bien pertenecen a una antigua tradición andina (Willey 1953), constituyen un rasgo que ha sido difundido de manera considerable por los Inkas (Raffino 1981).

\section{Características de la Piedra Hincada}

Este conjunto residencial se encuentra emplazado hacia el norte del sitio El Shincal de Quimivil, sobre el piedemonte oriental del Cerro Shincal (2305 msnm), a una altura de 1360 $\mathrm{msnm}$. A partir de los datos aportados por Raffino y colaboradores y de nuestras investigaciones sobre el terreno se establece que el conjunto se compone de cuatro espacios bien delimitados: a) tres de ellos, K.3, R.1 y un espacio cerrado intermedio (4,30 m por 4,20 m)2, alineados en sentido SO-NE; y b) un patio común a los anteriores de una superficie aproxi- 


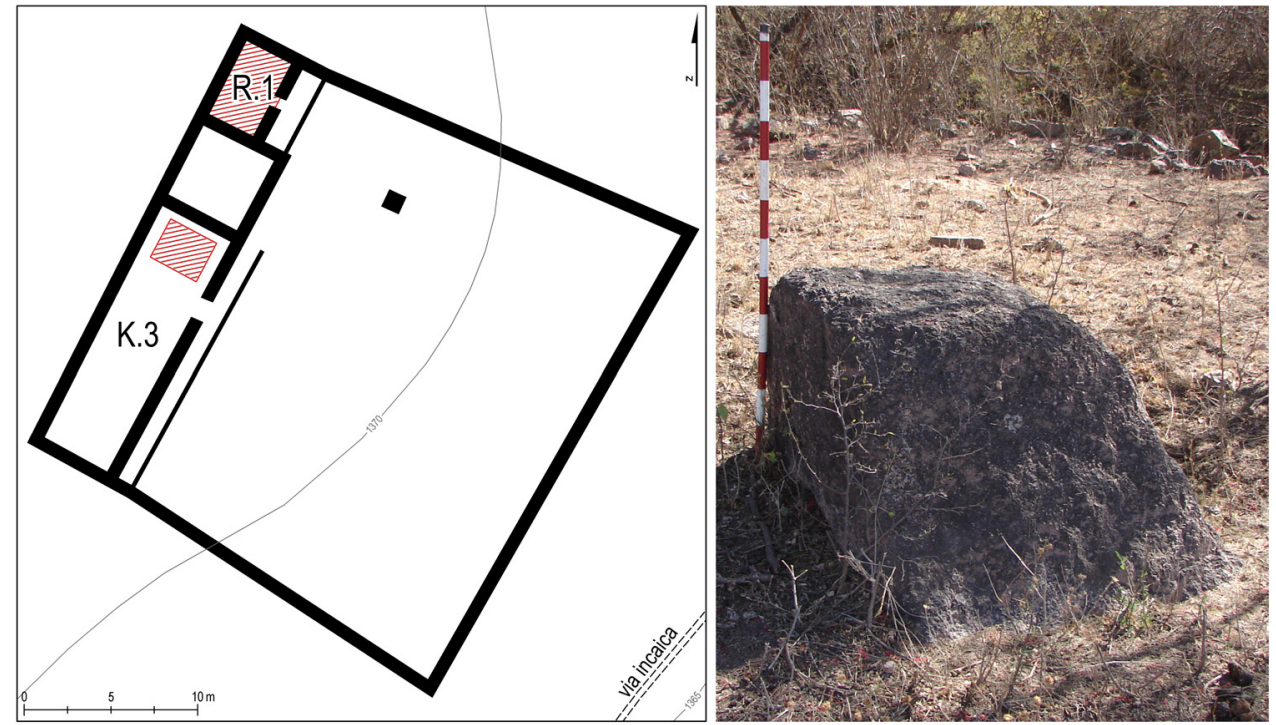

Figura 2. Izquierda: esquema general de la kancha Piedra Hincada mostrando los sectores excavados. Derecha: bloque de granito que domina el paisaje interior de la kancha (cada división del jalón representa $10 \mathrm{~cm}$ )

mada de $600 \mathrm{~m}^{2}$ y una pendiente NO-SE del 14\% (Figura 2). Farrington (1999) se refiere a este conjunto de tres recintos como kallanka, lo cual conduce a pensar en diferentes eventos constructivos (e.g. tabicamiento interno) a través del tiempo.

El recinto K.3 mide $14,75 \mathrm{~m}$ por 4,20 $\mathrm{m}$ y posee un vano de acceso de un metro de ancho con un escalón de piedra; sus muros son dobles de un espesor de $0,80 \mathrm{~m}$. Por su parte, el recinto R.1 posee $4,30 \mathrm{~m}$ por $2,80 \mathrm{~m}$, con un vano de acceso de $0,60 \mathrm{~m}$ de ancho y muros dobles de $0,60 \mathrm{~m}$ de espesor.

Un detalle interesante en la fachada de la Piedra Hincada es la existencia de una especie de banqueta o muro de una sola hilera de piedras. El mismo se encuentra sobre agregado a manera de contrafuerte a lo largo de K.3 y R.1.

Las paredes de la kancha están confeccionadas con rocas de granito canteadas y altamente seleccionadas provenientes de los cerros vecinos. Sobre la esquina noroeste del patio yace un bloque de granito de color negro. Posee una forma trapezoidal de 0,54 $\mathrm{m}$ de altura, 0,85 $\mathrm{m}$ en su base inferior y 0,56 $\mathrm{m}$ en su base superior (véase Figura 2). A partir de esta roca surge la denominación del conjunto Piedra Hincada. Según Farrington se trataría de “... a small, unworked black boulder just in front of a kallanka in its patio which this may well represent a secondary usnu stone (these are found occasionally in the Cusco area and are often unworked)" (Farrington 1999: 61). Para dicho autor, éste sería un elemento más que le confieren a El Shincal el carácter de "Nuevo Cusco".

\section{El Qhapaq Ñan en la Piedra Hincada}

La vía presenta una particularidad relacionada con la disposición de las rocas en sus bandas laterales: a) por un lado, observamos rocas canteadas y/o seleccionadas, dispuestas tanto a la derecha como a la izquierda de la vía, con sus caras planas hacia el interior. El espacio entre las rocas es constante, presentan un tamaño semejante y se pueden hallar 
enfrentadas entre sí o de manera alternada. Correspondería a una variedad de camino de "tipo despejado y amojonado" (Moralejo 2011); b) por otro lado, podemos observar una hilera de piedras dispuestas sobre uno de los laterales de la vía, en este caso sobre el borde ladera arriba. Formaría "una especie de muro de una sola hilada" (Vitry 2000: 191). Sus caras internas y externas están canteadas, mientras que la superior se mantiene lisa (no en todos los casos) y dispuesta paralelamente al suelo. Correspondería, también, a una variedad del tipo de camino "despejado y amojonado" tratándose, en este caso, de un alineamiento lateral contiguo de rocas hacia un solo lado del camino (Moralejo 2011).

Ya sea en uno u otro caso, se puede observar una forma de jalonamiento de la vía que responde a una determinada intencionalidad de los constructores inkas, razón por la cual entraría en lo que se clasifica como camino. Si a ello se le suma su relación con una de las kancha de la periferia del sitio, dicha intencionalidad podría estar vinculada con los movimientos de entrada y/o salida a El Shincal.

\section{La alfarería presente}

El material de excavación, parcialmente rescatado -como ya se dijo-, consistió principalmente en fragmentos de cerámica y proviene de dos sectores excavados en el Conjunto de la Piedra Hincada: uno de $3 \mathrm{~m}$ por 2,5 m sobre el lado norte del recinto K.3 y otro correspondiente a la totalidad del recinto R.1 (véase Figura 2). En ambos casos se siguieron los niveles estratigráficos naturales.

Se realizó un estudio cronotipológico de acuerdo al análisis de determinadas variables como: técnica de tratamiento, color, decoración, patrón del diseño y presencia/ausencia de rasgos utilitarios sobre ambas superficies (externa e interna); tipo de cocción; parte de la pieza; espesor; forma y caracterización de las pastas (Moralejo 2011). En el caso de K.3 se cuenta con un total de 60 fragmentos de cerámica y un resto óseo, procedentes de tres unidades estratigráficas. La asignación tipológica de los fragmentos de cerámica arrojó el siguiente resultado: Cerámica Tosca (43,33\%), Inka (31,66\%), Belén (15\%), Sanagasta (5\%) y Aguada-grupo grises (5\%). Por otra parte, R.1 posee 165 fragmentos de cerámica, un desecho de talla lítica de obsidiana y un tortero elaborado en cerámica Belén Negro sobre Rojo, procedentes de seis unidades estratigráficas a los que debe sumarse una recolección superficial intramuros. La asignación tipológica de los fragmentos de cerámica dio como resultado la presencia de Cerámica Tosca (50,30\%), Inka (45,45\%), Belén (3\%), Hispano-Indígena (0,60\%) y Ciénaga $(0,60 \%)$. El 1,21\% restante corresponde a fragmentos indeterminados.

En relación al remontaje y análisis morfológico de los fragmentos se determinó la presencia de diversas formas cerámicas como platos, platos ornitomorfos, aríbalos/aribaloides incaicos (entre los que se hallan imitaciones del Cuzco Policromo Rojo sobre Ante e Inka-Paya), escudillas del Tardío (e.g. Sanagasta, Belén) y vasijas globulares de cerámica tosca (Moralejo 2011)

\section{Discusiones}

La construcción de una kancha estaba supeditada a las condiciones particulares del terreno donde se erigía, la presencia y calidad de la materia prima y el interés y urgencia de sus constructores (Raffino 1981). Sean cuales fueran las características arquitectónicas adoptadas, este tipo de conjuntos, asociados a los caminos incaicos, constituyen un marcador cultural de alto valor simbólico. 
Como ya se dijo, la kancha Piedra Hincada forma parte del sitio El Shincal de Quimivil, un centro administrativo y religioso que tuvo un papel preponderante durante el incario. De acuerdo a los trabajos realizados en El Shincal, el patrón de asentamiento -organizado a partir de la gran plaza central (o aukaipata) y edificios circundantes- manifiesta una estructuración del espacio cuyo objetivo era lograr el desarrollo de las prácticas festivas fundamentales para mantener el esquema político del estado (Raffino 2004; Giovannetti 2009; Couso et al. 2011; Moralejo 2011). En relación a ello, resulta importante comprender el rol de la Piedra Hincada dentro del conjunto de actividades realizadas en el sitio.

El hecho de estar emplazada a la vera del camino, próxima a la plaza o aukaipata del sitio (véase Figura 1), y de contar con diversas formas cerámicas (platos, aríbalos/aribaloides, escudillas y vasijas globulares de cerámica tosca) de diferentes asignaciones tipológicas-estilísticas (Belén e Inkas) algunas de las cuales se asocian a procedencias no locales (Sanagasta) conduce a pensar que podría tratarse de residencias para determinados grupos de personas, algunos quizás provenientes de lugares distantes dentro del NOA. Dicha hipótesis se sustenta teniendo en cuenta la materialidad presente, en relación a la alfarería, en otros sectores del sitio como la Kancha II (Couso et al. 2011). Sin embargo, cabe señalar que haría falta más información como rasgos de quema, fogones, instrumentos líticos, restos óseos, semillas, carbón, entre otros, que permitan corroborar nuestra hipótesis sobre residencias domesticas. Asimismo, es necesario realizar estudios sobre evidencias de uso en los restos cerámicos para inferir actividades como la cocción y servido de alimentos, almacenamiento y transporte.

En relación al párrafo anterior, y debido a la significativa presencia de formas incaicas típicas como platos y aríbalos, también se plantea la posibilidad que el contexto registrado en la Piedra Hincada corresponda a actividades particulares de tipo ceremonial y/o ritual. Ello, según datos etnohistóricos y arqueológicos, podría estar relacionado con las prácticas festivas propiciadas por el Estado (Bray 2003). En este sentido la presencia del bloque trapezoidal de granito negro en el patio podría relacionarse con ese tipo de prácticas.

En cuanto a la exigua presencia de cerámica más temprana Ciénaga y Aguada, la misma podría deberse a procesos postdeposicionales propios del sitio (migraciones horizontales o verticales de los restos materiales a causa de agentes naturales y antrópicos). Igualmente, su mera presencia señala, como bien se sabe, la existencia de ocupaciones más tempranas o formativas en el área (González 1966, Raffino 2004).

Un dato a tener en cuenta es el posicionamiento de la Piedra Hincada dentro de la ruta principal de acceso a El Shincal de Quimivil. Ésta junto con la Kancha "y" conforman las primeras unidades residenciales, dentro del trayecto El Shincal de Quimivil - La Aguada, que ponen de manifiesto la relación de los transeúntes con el sitio (Moralejo 2011).

Para finalizar, es necesario continuar con las investigaciones de modo que las hipótesis delineadas puedan ser sometidas a mayor contrastación empírica y se pueda avanzar en la caracterización funcional y arquitectónica de este conjunto.

Agradecimientos: Al Dr. Rodolfo Raffino y a los Lics. Darío Iturriza, Diego Gobbo y Victoria Montes, como también a la Municipalidad de Londres, por conceder el material, planillas y libretas de los trabajos de campo. Asimismo quisiera agradecer al Consejo Nacional de Investigaciones Científicas y Técnicas, Agencia Nacional de Promoción Científica y Tecnológica, Facultad de Ciencias Naturales y Museo y Universidad Nacional de La Plata por el financiamiento de estas investigaciones durante mis trabajos de Tesis Doctoral. 


\section{Notas}

1 Las mismas dieron comienzo en el año 2005 a través de una beca de la Agencia Nacional de Promoción Científica y Tecnológica, Proyecto FONCyT: "NOA. Arqueología, Urbanismo, Ecología, Etnohistoria y Bioantropología Regionales" PICT Nº 13-10987. Posteriormente, continuaron a través de una Beca Doctoral y, recientemente, Postdoctoral del CONICET.

2 Este espacio cerrado intermedio posee mucho sedimento y clastos de derrumbe postdeposicional. En el mismo no se realizaron intervenciones arqueológicas (Iturriza, comunicación personal 2012).

\section{Bibliografía citada}

Bray, T.L.

2003 Inka pottery as culinary equipment: food, feasting, and gender in imperial state design. Latin American Antiquity 14(1): 3-28.

Couso, M.G., R.A. Moralejo, M.A. Giovannetti, L.M. del Papa, M.C. Páez, J. Gianelli, L.R. Giambelluca, M. Arnosio y R.A. Raffino

2011 Análisis de la variabilidad material del Recinto 1 - Kancha II: aportes para una comprensión de la política incaica en El Shincal de Quimivil. Arqueología 17: 35-55.

Farrington, I.S.

1999 El Shincal: un Cusco del Kollasuyu. Actas del XII Congreso Nacional de Arqueología Argentina (ed. por C. Diez Marín), Tomo I, pp. 53-62. Facultad de Ciencias Naturales y Museo, UNLP, La Plata.

Gasparini, G. y L. Margolies

1977 Arquitectura Inka. Centro de Investigaciones Históricas y Estéticas, Facultad de Arquitectura y Urbanismo, Universidad Central de Venezuela, Caracas.

González, A.R.

1966 Las ruinas del Shincal. Actas del Primer Congreso de Historia de Catamarca, Tomo III, pp. 15-28. Junta de Estudios Históricos de Catamarca. San Fernando del Valle de Catamarca, Argentina.

Giovannetti, M.

2009 Articulación entre el sistema agrícola, sistema de irrigación y áreas de molienda como medida del grado de ocupación Inka en El Shincal y Los Colorados (Valle de Hualfin, Provincia de Catamarca). Tesis Doctoral inédita No 1023. Facultad de Ciencias Naturales y Museo, Universidad Nacional de La Plata, Argentina.

Herrera, A.

2005 Las kancha circulares: espacios de interacción social en la sierra norte del Perú. Boletín de Arqueología PUCP 9: 233-255.

Hyslop, J.

1984 The Inka Road System. Institute of Andean Research New York, Academic Press, Inc. New York.

1990 Inka Settlement Planning. University of Texas Press, Austin. 
Madrazo, G. y M. Ottonello de García Reinoso

1965 Arqueología del Noroeste Argentino. Algunas interpretaciones funcionales para el período Tardío. ETNIA 2: 17-19.

Matos Mendieta, R.

1994 Pumpu. Centro Administrativo Inka de la Puna de Junín. Editorial Horizonte, Lima.

Moralejo, R.A.

2011 Los Inkas al sur del Valle de Hualfin: organización del espacio desde una perspectiva paisajística. Tesis Doctoral No 1150 . Facultad de Ciencias Naturales y Museo, Universidad Nacional de La Plata, Argentina. Disponible: http://sedici.unlp.edu.ar/handle/10915/5242

Raffino, R.A.

1981 Los Inkas del Kollasuyu. Ediciones Ramos Americana, La Plata, Argentina.

2004 El Shincal de Quimivil. Editorial Sarquís, San Fernando del Valle de Catamarca, Argentina.

Raffino, R.A., R.J. Alvis, L.N. Baldini, D.E. Olivera y M.G. Raviña

1982 Hualfín-El Shincal-Watungasta. Tres casos de urbanización Inka en el N.O. argentino. Actas del IX Congreso Nacional de Arqueología, pp. 470-497. Sociedad Chilena de Arqueología. Museo Arqueológico de La Serena. La Serena, Chile.

Vitry, C.

2000 Aportes para el estudio de caminos incaicos. Tramos Morohuasi - Incahuasi. Salta, Argentina. Editora Gofica. Salta, Argentina.

Willey, G.R.

1953 Prehistoric settlement patterns in the Virú Valley, Perú. Smithsonian Institution, Bureau of American Ethnology, Bulletin 155, Washington. 
